\title{
Measuring and Increasing Z-Width with Active Electrical Damping
}

\author{
David W. Weir* \\ J. Edward Colgate ${ }^{\dagger}$ \\ Department of Mechanical Engineering \\ Northwestern University \\ Evanston, IL 60208, USA
}

Michael A. Peshkin

\begin{abstract}
The "Z-Width" of a haptic display is the dynamic range of impedances that can be passively rendered. Haptic displays with larger Z-width generally render more realistic feeling virtual environments.

We present a new method for measuring and displaying the Zwidth of a haptic display. Instead of stiffness-damping plots, we believe a more illustrative technique for plotting the Z-width of a haptic interface is the envelope of achievable passive impedances as a function of frequency. Both hardware and analysis software for this new type of Z-width measurement are discussed.

As previous research has shown, the maximum passive impedance that a device can render is directly related to the physical damping available in the mechanism. In an effort to maximize the Z-width of the haptic display, we present a new technique for adding physical damping to a haptic display through the use of ana$\log$ electronics in the motor amplifier. Due to its electrical nature, active electrical damping has the benefit of dynamically variable parameters with no added mechanical complexity or mass.

With the addition of active electrical damping, we show a performance improvement via a larger $\mathrm{Z}$-width and larger range of passive virtual environment parameters.
\end{abstract}

Index Terms: H.5.2 [User Interfaces]: Haptic I/O-Haptic Displays; H.5.2 [User Interfaces]: Haptic I/O-Rendering; H.5.2 [User Interfaces]: Haptic $\mathrm{I} / \mathrm{O}-\mathrm{Z}$-width

\section{INTRODUCTION}

Haptic displays are devices that generate mechanical impedances. We refer to "impedance" here as a dynamic relationship between force and velocity.

The "Z-Width" of a haptic display is the dynamic range of impedances that can be passively rendered. The Z-width of a haptic display is of concern because, generally speaking, haptic displays with larger Z-width render better feeling virtual environments. It is desirable therefore to maximize the $\mathrm{Z}$-width of a haptic display. Our common experience of interacting with ordinary objects in the physical world exposes us to widely varying impedances, ranging from almost zero when moving through free space, to extremely high when interacting with rigid objects, such as walls and floors. An ideal haptic display would render this same dynamic range of impedances, however practical haptic displays are limited in their $\mathrm{Z}$-width. The goal of a haptic interface designer is to maximize the capability and dynamic range of the device.

We present a new method for assessing the Z-width of a haptic display that shows a more complete picture of the behavior as a function of frequency.

*e-mail: d-weir@northwestern.edu

†e-mail: colgate@northwestern.edu

†e-mail: peshkin@northwestern.edu

Symposium on Haptic Interfaces for Virtual Environments and Teleoperator Systems 2008

13-14 March, Reno, Nevada, USA

978-1-4244-2005-6/08/\$25.00 @2008 IEEE
Passivity is a well established framework for analyzing the stability and performance of haptic displays. The basic definition of passivity is that the energy extracted from a system cannot exceed the initial energy in the system. This is typically determined by integrating the power produced over time, where power is defined as the product of force and velocity for translational mechanical systems. A passive haptic display cannot supply energy to the user. The advantage of using passivity as a benchmark is that a passive system, coupled with any other passive system, is necessarily stable. Humans are typically considered passive, so if a haptic display can be guaranteed passive, then the coupled system will also be stable when the human interacts with the haptic interface. Colgate and Schenkel and also Adams and Hannaford present more rigorous explanations of passivity and coupled stability in [5] and [1]. The use of passivity also avoids the problem of repeatedly analyzing and predicting the stability of the coupled system for varying rendering parameters and user interaction behaviors.

As previous research has shown, the maximum passive impedance that a device can render is directly related to the physical damping available in the mechanism. In Section 4, we present a method of adding physical damping to a haptic display through the use of analog electronics in the motor amplifier. With this addition of active electrical damping, we show a performance improvement via a larger $\mathrm{Z}$-width and wider range of passive virtual environment parameters.

Impedance, as a dynamic relation between force and velocity, is typically considered to define the resulting force provided by a haptic display for a given velocity input. Equation 1 is a standard definition of impedance. Admittance is the inverse of impedance. Admittance causality assumes the force applied to the system is the input and the resulting velocity is considered the output.

$$
Z(s)=M s+B+\frac{K}{s}
$$

\section{Z-WIDTH}

"Z-Width" is defined as the dynamic range of passive impedances that can be rendered by a haptic display. There are two aspects of Zwidth that specifically deserve emphasis. The first is passivity, as it is a well developed framework from which to address the questions of stability and robustness. The second is the frequency dependent nature of both impedance and passivity that a discussion of Z-width addresses. That is, Z-width implies the dynamic response of the system over a broad range of frequencies.

Typically, Z-width is illustrated by plotting the region of passive virtual environments using stiffness and damping ( $\mathrm{K}$ and $\mathrm{B}$, respectively) as the axes. The line in the K-B plane indicates the region of stability; the area under the curve comprises virtual environment parameters that result in stable virtual wall collisions. See for example, Colgate and Brown and also Mehling et al. for more detailed information regarding KB-type plots and analysis of system performance [4], [12]. This method is useful for illustrating differences in performance between virtual environments on a single haptic display, but it is less useful for directly comparing different haptic displays. One of the primary limitations of KB-type plots is the lack of 


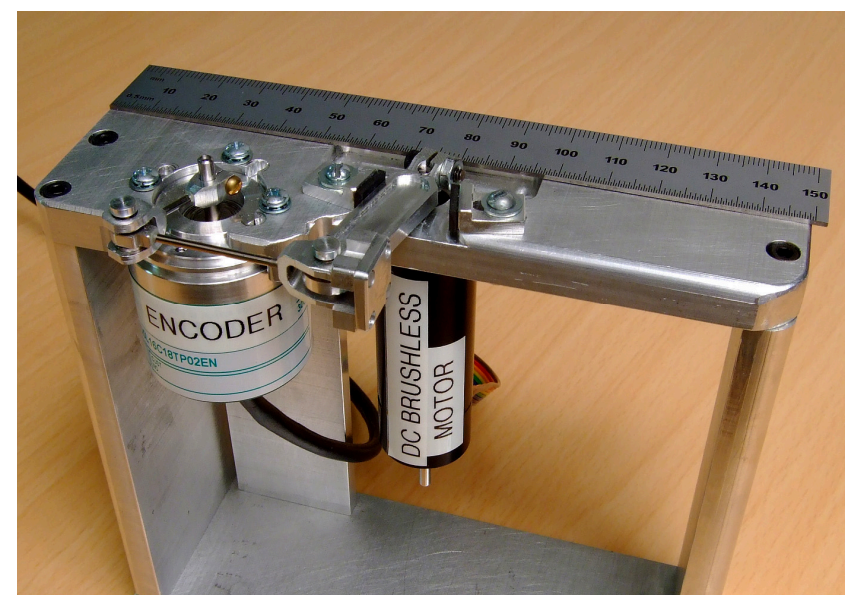

Figure 1: Photograph of haptic display used for these experiments, with ruler providing a size reference.

information regarding the mass of the haptic display and the virtual mass being rendered. Another limitation is the lack of explicit frequency information. Since KB-type plots are typically generated using virtual wall collisions, a wide range of frequency information is captured but not clearly displayed. If one were to compare two different haptic displays, what would be the appropriate metric? Would it be the maximum passive impedance, the bandwidth of a haptic display when rendering zero added mass, the Z-width, or some other metric? Consider the following scenario: if one were to take two identical haptic interfaces and bolt their endpoints together, then the maximum achievable stiffness and damping should be twice as high as those of a single display. The KB-type plot of the coupled system would show a marked improvement. But, has performance really improved, or is this simply a larger device? The minimum expected stiffness and mass would also be twice as large in the coupled device scenario.

Another technique for showing Z-width was presented in Adams et al., where the extremes of admittance and impedance were plotted as a function of frequency [2]. This technique used a mathematical model of the haptic display with a virtual coupling. The virtual coupling parameters were selected to ensure stability of the combined system. A plot was generated showing the system model as well as theoretically and experimentally derived passive virtual coupling parameters. This illustrates the performance of the device, although does not clearly illustrate some of the practical implementation limitations of performance, such as friction and delay, except through the deviation between experimental virtual coupling parameters and theoretically predicted limits.

To address these issues, we present a new way of plotting Zwidth information, that is to display a set of frequency response curves. Specifically, the envelope of passive impedances as a function of frequency that can be rendered by a haptic display. In this way, behavior may be more clearly characterized and compared across devices.

The haptic display used for these experiments is a small, highfidelity device with low peak force and high resolution position sensing, as shown in Figure 1. The apparent mass of the haptic display at the endpoint is approximately 5 grams, with an endpoint position sensing resolution of 0.5 micrometers, a maximum display force of approximately 8 Newtons, and a virtual environment servo rate of $1 \mathrm{kHz}$. Note that a physical damper is not present in the mechanism and a brushless motor is used. Weir et al. provides a more detailed description of the haptic display [14].

For illustration, Figure 2 is a KB-type plot with zero added vir-

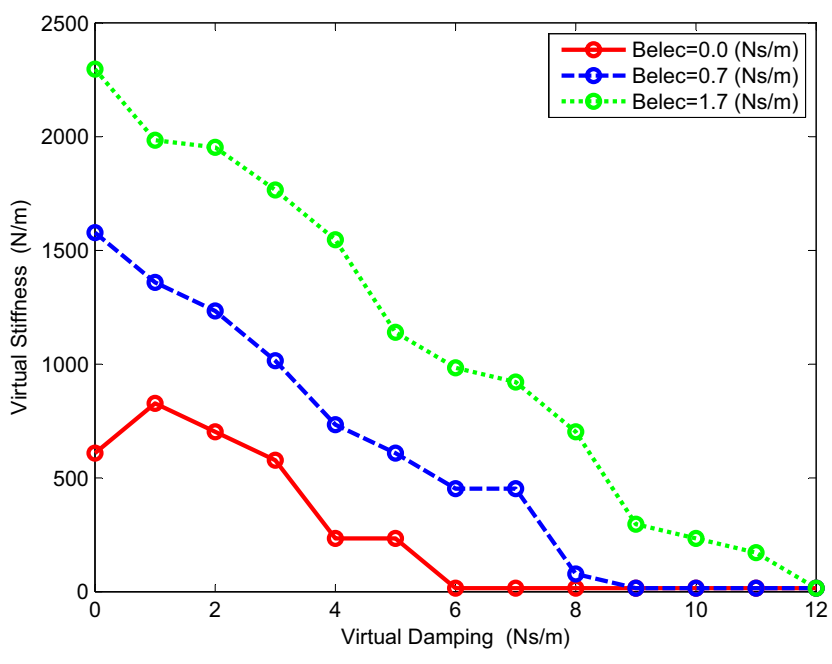

Figure 2: Standard stiffness-damping plot of haptic display while rendering a virtual environment mass of zero grams. The area under each curve indicates stable virtual wall parameters.

tual mass for our haptic display. Figure 3 is a Z-width plot showing the envelope of passive impedances over the frequency range of the device.

By showing the impedance of the haptic display as a function of frequency, it is possible to observe how the behavior changes, such as spring-like behavior at low frequencies and mass-like behavior at higher frequencies, in addition to mechanical resonances. The overall Z-width plot is composed of a series of curves from individual trials, each with a specific combination of virtual environment impedance parameters: virtual stiffness, virtual damping, and virtual mass. Each curve has a specific shape, corresponding to the actual behavior of the haptic interface while it is rendering the virtual impedance. The envelope, comprising both magnitude and phase, is determined by calculating the maximum and minimum passive impedance at every frequency point. That is, the $\mathrm{Z}$-width envelope is iteratively updated as successive trials are performed, as each data set is analyzed.

Figure 4 shows the dynamic response of the haptic display for an individual trial; the magnitude and phase of the experimentally measured impedance are plotted while the haptic display is rendering a virtual environment. The virtual environment parameters for this trial are a virtual stiffness, $K$, of 100 Newtons per meter, a virtual damping, $B$, of 1 Newton seconds per meter, and a virtual mass, $M$, of 0.010 kilograms. The virtual environments used in this set of experiments are parameterized with $K, B$, and $M$. The virtual stiffness is implemented using the raw position data from the encoder. Velocity information for the virtual damping is obtained by differentiating the position signal and then passing though a 100 Hertz second order digital filter. Acceleration data for virtual mass rendering is similarly obtained by differentiating the position twice and then filtering with a 72 Hertz second order digital filter. The three virtual forces from stiffness, damping, and mass are summed to produce the net force commanded to the haptic display. The Zwidth of our haptic display is shown here for this particular class of controllers parameterized by $K, B$, and $M$. There is no guarantee that another controller design, such as different velocity filters, would not extend the Z-width.

The ideal Z-width envelope comprises all possible passive trials within the space of virtual environment parameters. We relied on an initial grid-based search in the parameter space, and then subsequently performed more trials in areas of interest to expand the Z-width and capture the limits of passivity. Unfortunately, a grid- 

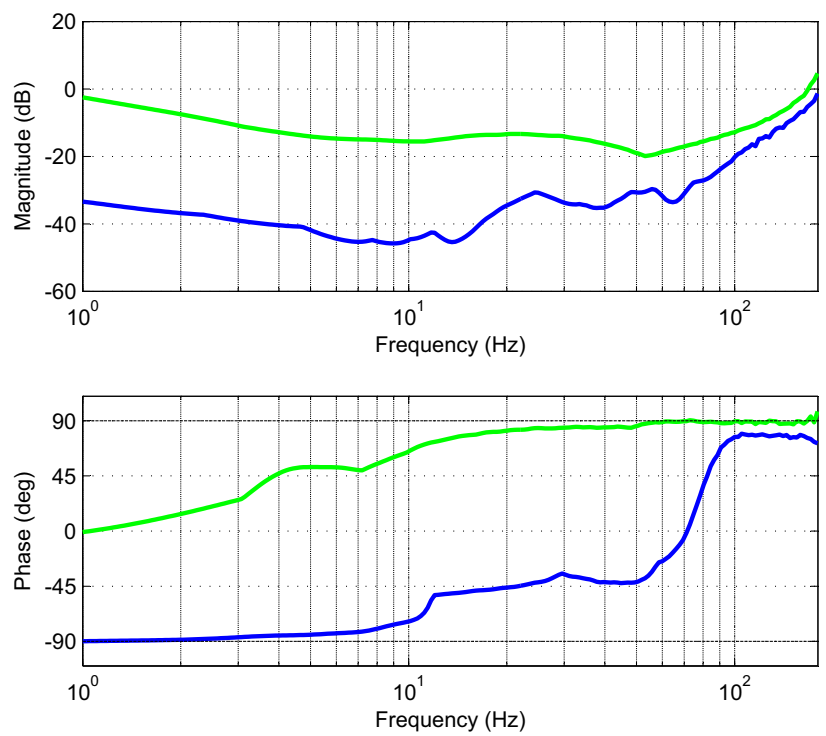

Figure 3: Z-Width of haptic display with no additional damping present. The lines indicate the maximum and minimum value at each frequency for both the impedance magnitude envelope and phase envelope.
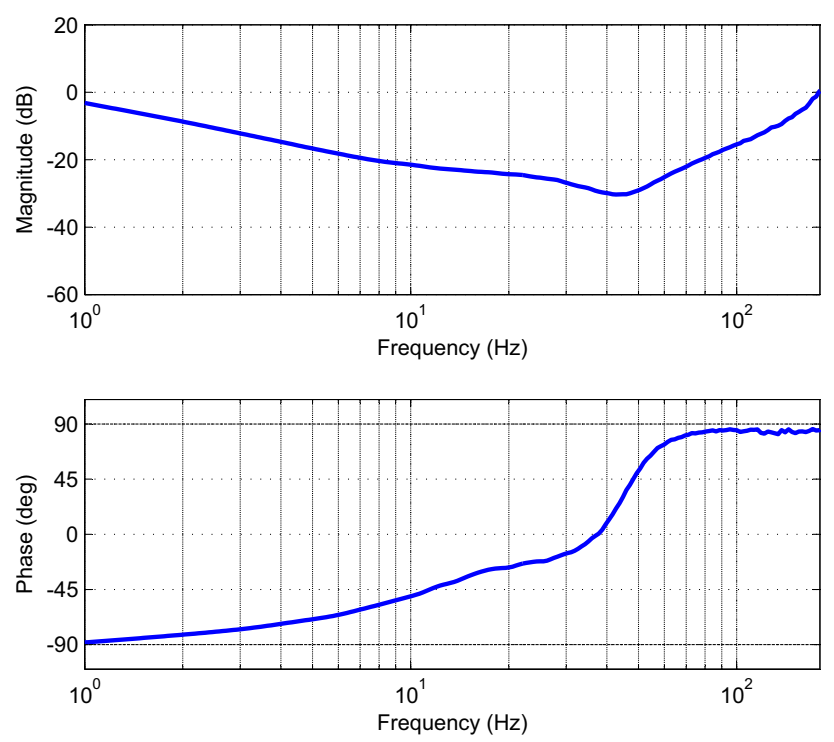

Figure 4: Impedance plot of haptic display dynamic response. Trial virtual environment parameters: $K=100 \mathrm{~N} / \mathrm{m}, B=1 \mathrm{Ns} / \mathrm{m}, M=0.01$ $\mathrm{kg}$. based search through $K, B$, and $M$ space results in an exhaustive set of parameters, rather than a more efficient directed search. More efficient search algorithms is a subject for future research.

\subsection{Z-Width Measurement}

The impedance of the haptic display is measured by applying an external disturbance torque and measuring the resulting velocity of the haptic display while it is rendering a virtual environment.

The fidelity of the measured velocity signal is important, due to the wide frequency range of velocity information desired for analysis and the typically small deflections and velocities from the external perturbation. In our case, due to the design of the haptic display used for this experiment, in which the motor and the position sensing encoder are not colocated, we added a velocity sensor to the end of the motor shaft. On more typical haptic displays, one might use the position sensor of the haptic display to provide velocity measurements, however low encoder resolution and low sampling rates can cause poor velocity measurements, so caution must be exercised when taking that route.

The velocity sensor consists of a small voice coil motor used in reverse; the coil is attached to the haptic interface motor shaft, which causes it to move through the magnetic field of the stator, generating a voltage. The velocity sensing coil is connected to an instrumentation amplifier, adding an extremely high resistance (approximately $10^{10} \Omega$ ) in series with the coil and preventing current from flowing through the voice coil. Any current flowing though the velocity sensing coil would create an undesirable torque. The induced voltage, the velocity signal, is amplified by the instrumentation amplifier before sampling and analog to digital (A/D) conversion. The $\mathrm{Z}$-width velocity sampling and $\mathrm{A} / \mathrm{D}$ conversion are performed at a higher rate $(4 \mathrm{kHz})$ than the haptic display sample rate $(1 \mathrm{kHz})$ and use a separate data acquisition system from the data acquisition system used to control the haptic display, due to hardware limitations of the particular system. Although the higher sampling rate is required, the separate data acquisition system was used for convenience. Note that this sensor directly measures velocity, not position, therefore no differentiation is needed.

To generate external disturbance torques, another small voice coil motor is attached to the velocity sensor. The perturbation torque must be independent of the torque from the virtual environment. The torque motor uses a separate linear current amplifier from the haptic display. The disturbance torque command is amplified and the actual current flowing through the voice coil is measured. This measured current signal is converted to torque and is the torque input signal used for calculating the Z-width.

The resulting motor and velocity sensor comprise a $\mathrm{Z}$-width "probe" used to characterize the haptic interface, shown in Figure 5. In order to minimally alter the dynamics of the haptic interface, the $\mathrm{Z}$-width probe was designed to have very low rotational inertia, increasing the apparent inertia of the haptic display by approximately 3 grams. This tool allows us to analyze the behavior of the haptic display.

\subsection{Analyzing Impedance}

One key aspect of determining Z-width is to measure the behavior and passivity over a wide range of frequencies. By characterizing the device over its useful frequency range, one can ascertain the device performance; this also makes limitations apparent, such as mechanical resonances. To capture device performance over a wide range of frequencies, a white noise signal was selected as the perturbation torque. Instead of repeatedly testing every set of virtual environment parameters using a series of sinusoidal torque inputs at varying frequencies, the white noise input had the advantage of performing more of a continuous type of test as a function of frequency. This means frequency gaps in which isolated and important behavior could fall are unlikely. Another advantage of 


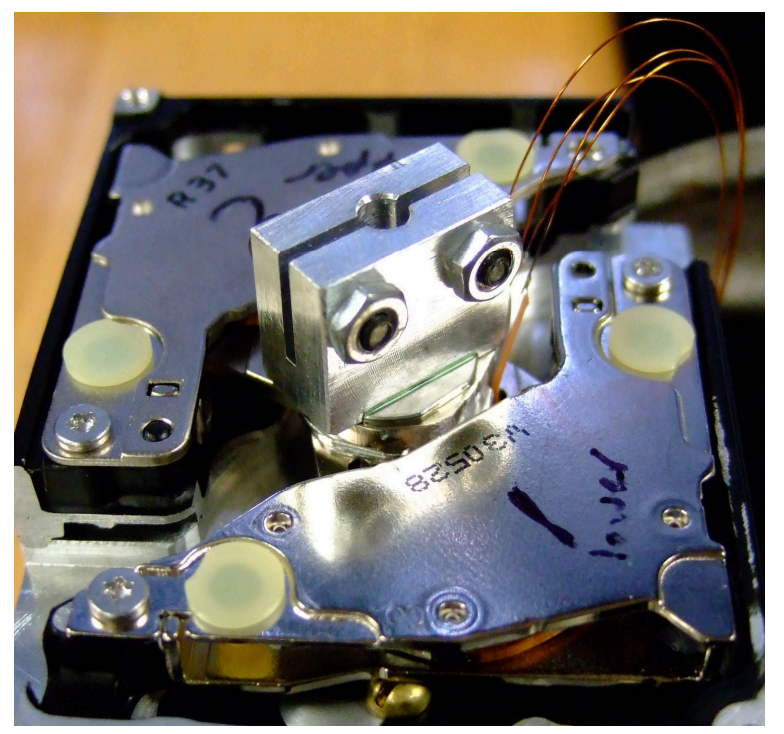

Figure 5: External Z-width probe to measure performance of haptic display. For size reference, the length of the long axis of the clamping block is approximately $10 \mathrm{~mm}$.

the white noise input is that it requires a single long trial for each set of impedance parameters, instead of repeated tests at different frequencies, making data collection more efficient.

The torque and velocity data are post-processed in order to calculate the impedance of the haptic display for each set of virtual environment parameters. In our case, the selected length of each data set was 90 seconds of input sampled at $4 \mathrm{kHz}$. After collection, the data was detrended to remove any constant offset or linear drift over the length of the data set.

The analysis was performed using Matlab with the SPA spectral analysis algorithm. Using the velocity as the input and the torque as the output, an estimate of the transfer function, representing the impedance of the haptic display, is calculated. One such trial is shown in Figure 4. The SPA function uses a series of overlapping windows to reduce noise in the system estimate, effectively filtering the result. There is a trade-off between detail, specifically frequency resolution, and noise in the analysis. We found a window length of 2048 points to be a reasonable compromise between detail and a noise for 90 second data sets sampled at $4 \mathrm{kHz}$. The amount of noise also depends on ratio of window length to data set length. Due to the windowing and detrending of the data, postprocessing is required; given the chosen analysis procedure it is not possible to calculate the impedance in real time.

\section{Maximizing Z-WIDTH}

To improve the performance of a haptic display, it is desirable to expand the Z-width. There are two general methods to address the issue of dynamic range. The first is to directly increase the impedance range that can be passively rendered by the haptic display. The second general method is to take advantage of psychophysical techniques to alter the user's perception of the dynamic range of a haptic display. We will not address psychophysical techniques in this paper, but some examples include the work of Lawrence et al. in rate-hardness to increase the perceived stiffness of virtual walls, Okamura et al. adding open loop vibration feedback to improve the realism of contact, and Kuchenbecker et al. extending open loop vibration to event-based methods that mimic the transient characteristics of interaction [11], [13], [10].

The minimum impedance that can be passively rendered is largely dictated by the mechanical, sensing, and feedback design of the haptic display. Since lowering the minimum bound is a less accessible target, most research efforts, ours included, focus on increasing the maximum impedance that can be rendered. At the high impedance limit of a haptic display, the parameters of the virtual environment, i.e. the mass, damping, and stiffness, are all coupled in their effect on passivity. Colgate and Schenkel show that the maximum stiffness that can be rendered while maintaining passivity is directly affected by the level of physical damping present in system [5].

$$
b>\frac{K T}{2}+B
$$

Where $b$ represents the physical damping in the mechanism, $K$ is the virtual environment stiffness, $T$ is the sampling interval in seconds, and $B$ is the virtual environment damping. This inequality leads to the conclusion that to increase the maximum stiffness that can be rendered one needs to add physical damping to the mechanism. Adding mechanical dampers to a haptic display is quite effective, as illustrated by Colgate and Brown, although there are also some negative aspects of mechanical dampers such as increased mechanical complexity, friction in the required seals, and added mass [4]. Passive electrical damping is an attractive alternative, as demonstrated by Mehling et al. [12]. There are no added mechanical components, and because it is implemented by an electrical circuit the damping parameters can be changed dynamically.

Unfortunately, previous work has shown that without specifically selected components, passive electrical damping is limited in the performance that it can provide. The maximum attainable level of passive electrical damping occurs when the motor rotor is shortcircuited or "crowbarred;" this presents a problem in that the motor cannot be driven while crowbarred [12].

$$
b_{e q}=\frac{K_{m}^{2}}{R_{m}}
$$

Equation 3 is the maximum equivalent mechanical damping provided by a crowbarred motor, where $K_{m}$ is the motor torque constant (also the back EMF constant), and $R_{m}$ is the rotor winding resistance. For our haptic display, this level of damping is extremely small, equal to $1.7 \times 10^{-4}$ Newton meter seconds per radian or in translational units, $3.7 \times 10^{-5}$ Newton seconds per meter. This low level of damping limits the applicability of passive electrical damping when using typical haptic display motors.

\section{Active Electrical Damping}

We developed active electrical damping to address the limitations of passive electrical damping. Active electrical damping provides a continuous velocity signal by means of an analog circuit, which is then scaled and added to the torque command provided by the haptic display. The electrical damping and current amplifier for the haptic display are implemented entirely using analog circuitry. There is no physical damper present in the haptic display used for these experiments.

Because passive electrical damping is limited by the resistance of the motor winding, we designed an analog circuit to circumvent the limitation of the rotor resistance. However if only the resistance were compensated for, the inductance of the motor winding would remain. The winding inductance can create large voltages when the motor is moving rapidly or, a more common occurrence in haptic displays, when the current changes rapidly such as when the haptic display collides with an object in the virtual environment. Therefore, the inductance of the motor must also be compensated for to provide an accurate velocity estimate.

We would like to add physical damping to the motor, represented by $b$ in Equation 4, artificially increasing $b$. It is also desirable to control the added damping, such as turning it on when needed for passivity and off otherwise, or varying the level of added damping. 


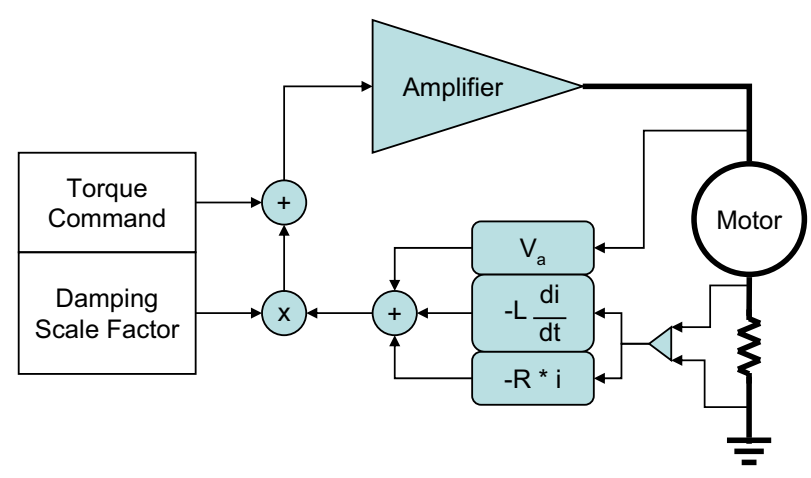

Figure 6: Block diagram of analog electrical damping, torque command, and motor amplifier. The highlighted items are implemented using analog electronics, while the torque command and scale factor for added damping are outputs from the discrete controllers.

$$
J_{m} \ddot{\theta_{m}}+b \dot{\theta_{m}}-\tau_{\text {ext }}=K_{m} i
$$

Equation 5 is the standard electrical governing relation for a motor, where $L$ represents the inductance of the winding, $i$ is the current flowing through the winding, $R_{m}$ is the winding resistance, $K_{E M F}$ is the back EMF constant of the motor, which is also equal to the torque constant of the motor, $\dot{\theta}$ is the angular velocity of the rotor, and $V_{a}$ is the voltage applied to the motor by the amplifier.

$$
\begin{gathered}
L \frac{d i}{d t}+R_{m} i+K_{E M F} \dot{\theta}=V_{a} \\
\frac{V_{a}-R_{m} i-L \frac{d i}{d t}}{K_{E M F}}=\dot{\theta}
\end{gathered}
$$

To add damping to the system, an analog circuit computes velocity by solving for the back EMF voltage of the motor, illustrated in Equation 6, a straightforward manipulation of Equation 5. The voltage applied to the motor is measured, and the calculated inductance, $L \frac{d i}{d t}$, and resistance, $R_{m} i$, are subtracted from the applied voltage, leaving a signal proportional to the back EMF and the velocity. This velocity signal is then scaled by multiplying it with a damping scale factor command signal. The resulting damping signal is then added to the torque command and the combined signal is commanded to the motor current amplifier.

The motor current is measuring using a current sensing resistor $(0.05 \Omega)$ and instrumentation amplifier. Four-quadrant analog voltage multipliers (Analog Devices model AD534) are used to multiply signals, while differentiation, inversion, addition, and scaling are accomplished using operational amplifiers. Analog electronics were used due to the extremely high bandwidth available and lack of sampling, providing continuous information. Of course, noise and oscillation are typically more of a problem with analog circuitry than digital implementations, and our circuit is no exception. The maximum level of damping that can be added to the system is limited by the stability of the active damping circuit.

Figure 6 shows a block diagram on the analog circuit used to implement active electrical damping, computing the velocity of the motor rotor. The circuit provides a continuous signal representing velocity, which is used in feedback with the motor amplifier to provide physical damping to the system.

In order to accurately cancel the resistance and inductance of the motor winding, the active electrical damping circuit parameters must be carefully tuned. During operation, current flowing through the motor causes heating, due to the energy dissipation by the winding resistance. The resistance of the motor winding changes significantly with temperature, requiring compensation or active tuning to the damping circuit parameters. The thermal coefficient of resistance of copper is 0.00393 percent per degree Celsius, so as the winding increases from a nominal temperature of $20 \mathrm{C}$ to an potential operating temperature of $60 \mathrm{C}$, for example, the winding resistance increases by approximately 16 percent. To account for this potential parameter change, the temperature of the motor winding is measured during operation and used to tune the resistance loss calculation circuit.

One advantage of the active electrical damping circuit is that it is sensorless; no position or velocity sensors are needed to add damping to the system. The position encoder is utilized solely for rendering the virtual environment on the haptic display. All of the information needed to calculate the rotor velocity comes from the motor voltage and current.

This velocity estimation circuit is different from typical back EMF estimators, in that it works at the low angular velocities typical of haptic display motors. Typical sensorless brushless motor controllers work by finding the zero crossings of the back EMF signal as the rotor turns to infer rotor position and then commutating the windings appropriately for the calculated rotor position. Back EMF zero crossing velocity estimators work well for applications such as pumps and fans, but do not work for haptic interfaces, where the rotor is moving slowly and frequently stopping and changing direction.

Another advantage of active electrical damping is that is can be controlled dynamically. Since the level of damping provided to the system is controlled by a voltage command, the active electrical damping can be enabled and adjusted during operation of the haptic display.

Diolaiti and Niemeyer also utilize an analog circuit to cancel the winding resistance, however their goal is not to add damping to the mechanism, rather it is to provide a stiff coupling to the virtual environment [6]. They also use the circuit to implement a wave variable approach to controlling the haptic display.

Also related is the work of Kawai and Yoshikawa, in which the authors implement an analog circuit to control the stiffness and damping of each joint in a multi-degree of freedom haptic display [8], [9]. The result is similar to the effect of a virtual coupling in that the stiffness and damping values are limited to ensure that the resulting behavior of the haptic display is stable while interacting with objects both in the virtual environment and in the real world.

\section{REsULts}

Figure 7 shows the impedance of the haptic display rendering a virtual environment both with and without added active electrical damping. The virtual environment parameters are: $K=50 \mathrm{~N} / \mathrm{m}, B$ $=2 \mathrm{Ns} / \mathrm{m}, M=0 \mathrm{~kg}$ and are the same in both trials. The added physical damping, $1.74 \mathrm{Ns} / \mathrm{m}$, increases the impedance, visible in the upper, solid line of the added damping trial. Note that although there is little visible difference in the magnitude of the impedance at high frequencies, there is a significant phase advantage. The added damping moves the phase of the impedance away from the +90 degree passivity limit improving the passivity margin. This indicates that higher impedance virtual environments, for example higher virtual stiffnesses, may be rendered while maintaining passivity. It is also important to note that the actual performance of the haptic display may not reflect the commanded virtual environment parameters. In the previous example, the commanded virtual mass is $0 \mathrm{~kg}$, however no haptic display can accurately render zero mass. The actual performance deviates significantly from ideal, particularly at high frequencies where the actual mass of the haptic display becomes a dominant factor in the measured behavior, as indicated by the increasing slope at high frequency in Figure 7.

The Z-width of the haptic display with $1.74 \mathrm{Ns} / \mathrm{m}$ additional active electrical damping is shown in Figure 8. The peaks in the envelope near $100 \mathrm{~Hz}$ are due to a resonance created by the interaction 

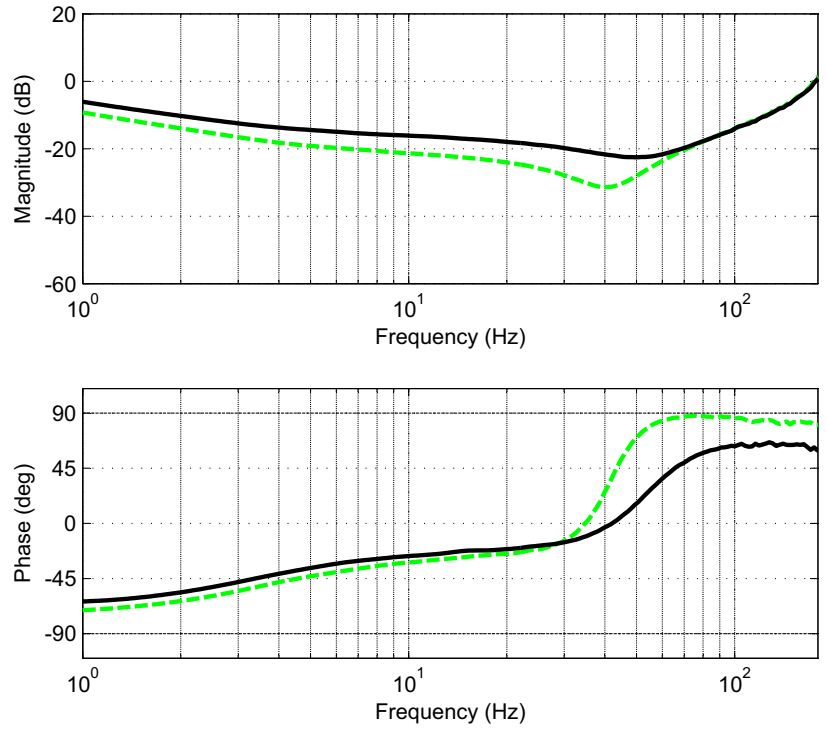

Figure 7: Performance of haptic display with (solid line) and without (dashed line) analog damping, while rendering virtual environment parameters: $K=50 \mathrm{~N} / \mathrm{m}, B=2 \mathrm{Ns} / \mathrm{m}, M=0 \mathrm{~kg}$. The level of added active electrical damping $=1.74 \mathrm{Ns} / \mathrm{m}$.
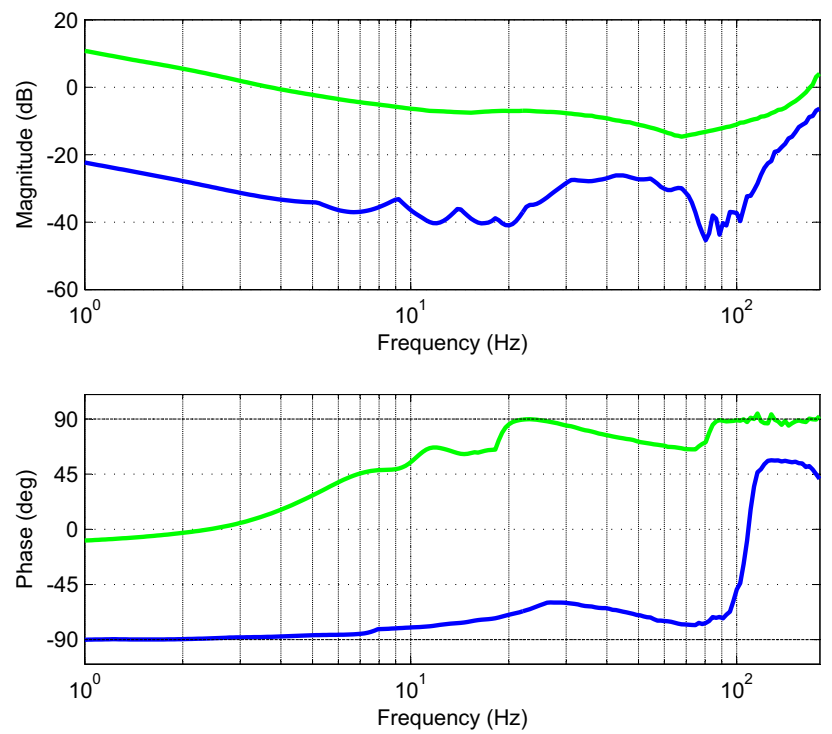

Figure 8: Z-width of haptic display with $1.74 \mathrm{Ns} / \mathrm{m}$ additional active electrical damping.

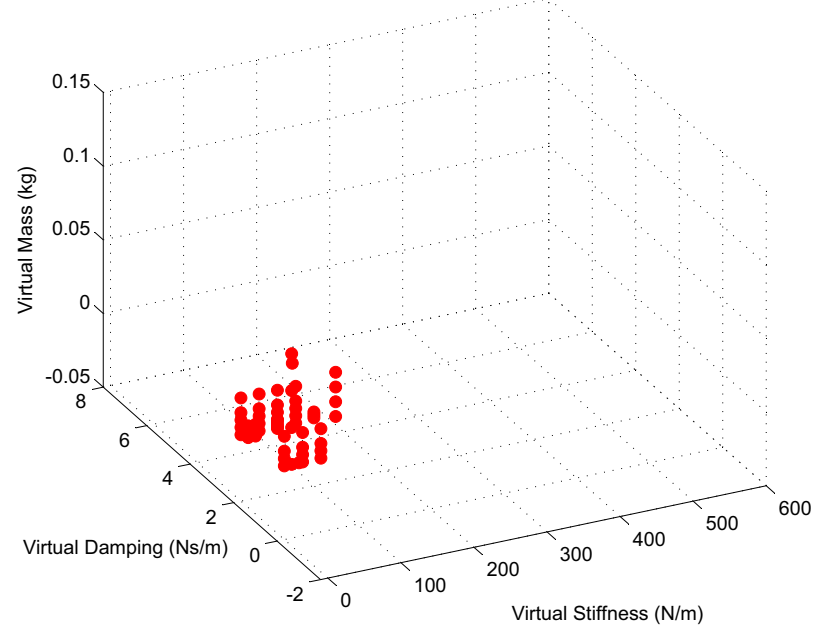

Figure 9: Passive virtual environment parameter space for haptic display without added damping.

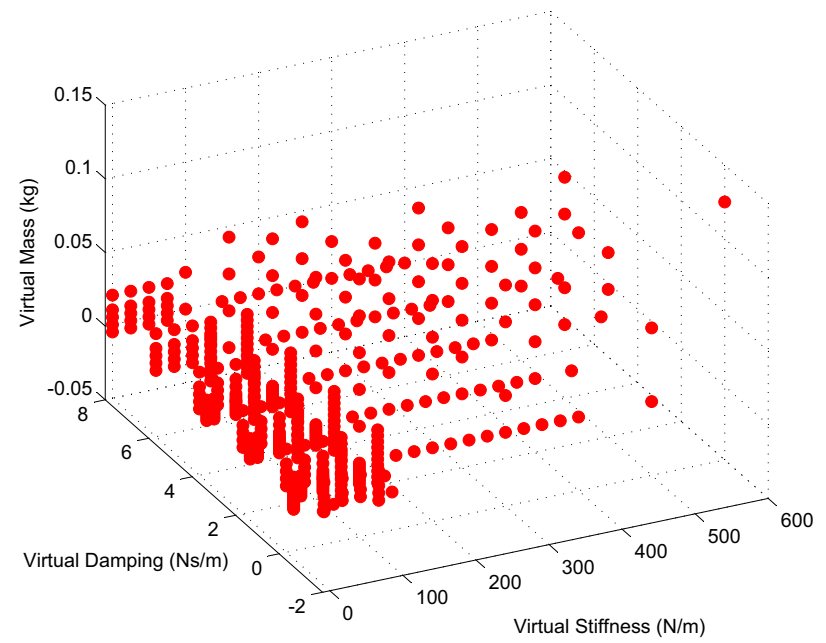

Figure 10: Passive virtual environment parameter space for haptic display with $1.74 \mathrm{Ns} / \mathrm{m}$ added active electrical damping.

of the virtual environment parameters, specifically when the virtual mass is negative, and the added electrical damping. These parameters resulted in non-passive behavior in the non-damped tests, but not in the experiments with added electrical damping.

The maximum passive virtual stiffness that can be rendered with the addition of active electrical damping increased from $100 \mathrm{~N} / \mathrm{m}$ to $600 \mathrm{~N} / \mathrm{m}$, visible as the upper bound of the Z-width envelope at low frequency in Figure 11. The maximum passive virtual damping increased from $3 \mathrm{Ns} / \mathrm{m}$ to $8 \mathrm{Ns} / \mathrm{m}$ with the addition of active electrical damping, and the minimum virtual mass that can be passively rendered decreased from $-0.008 \mathrm{~kg}$ to $-0.022 \mathrm{~kg}$. This performance improvement can likely be attributed to the additional damping in the mechanism providing dissipation to counteract energy leaks and other causes of non-passivity and instability [7], [5]. The passive virtual environment parameter space, in $K, B$, and $M$, without added damping is shown in Figure 9. Figure 10 shows the passive virtual environment parameter space with $1.74 \mathrm{Ns} / \mathrm{m}$ added active electrical damping.

Figure 11 shows the two Z-width envelopes plotted on the same axes. It is evident that primary benefit to added electrical damping 

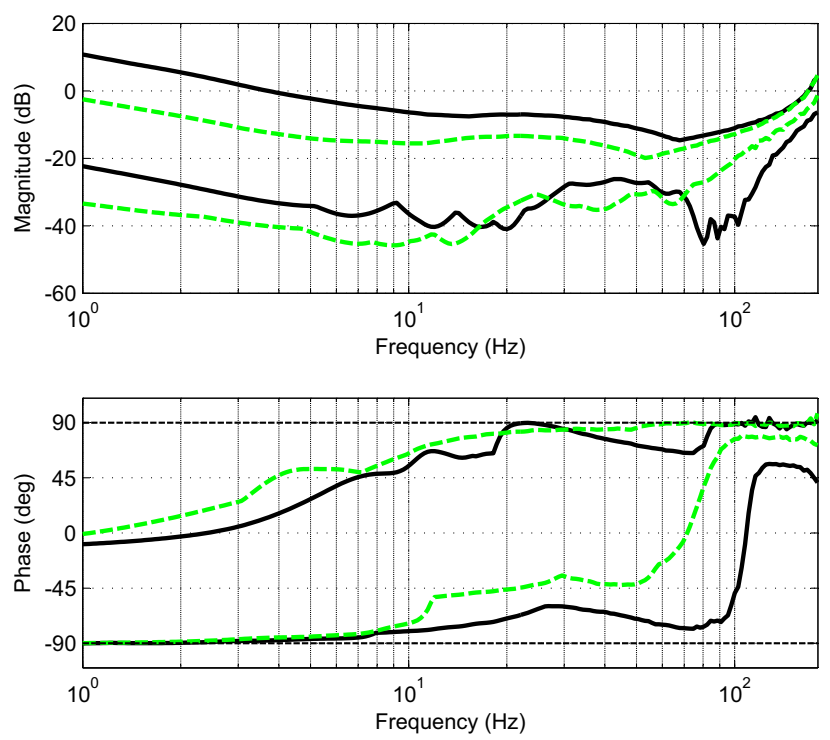

Figure 11: Z-width of haptic display for 2 cases: with added electrical damping (solid lines) and without (dashed lines).

is increasing the maximum passive impedance, indicating stiffer, more damped virtual environments can be passively rendered with the added dissipation.

\section{Conclusion}

We present a new method of displaying the Z-width of a haptic display which shows device performance as a function of frequency and shows the passive impedance limits. This method of displaying experimentally derived performance data captures more of the relevant information, such as frequency information and virtual environment mass that was difficult to discern from previous stiffnessdamping plots illustrating device performance and Z-width.

The Z-width probe effectively perturbs the haptic display and measures the resulting velocity, providing data to calculate the impedance of the system, while minimally changing the properties of the system under test. The Z-width probe is specifically adapted to work with the haptic display used here, however relatively simple modifications could be made to the design to permit characterization of other haptic interfaces.

In order to maximize device performance, specifically the dynamic range, it is desirable to add damping to the haptic display. We built an analog circuit to implement active electrical damping, improving the performance of the haptic display. With added active electrical damping, the interface can render virtual environments with approximately 6 times higher stiffness, 2.7 times more virtual damping, and can mask 2.75 times more inertia, while maintaining passivity. As can be seen in Figure 11, the primary effect is to increase the overall impedance of the haptic display, allowing a wider range of passive virtual environments. Future work will address adding negative virtual damping to counteract the additional physical damping in the system and shift the lower impedance boundary back toward the original undamped boundary.

Active electrical damping has a number of advantages, such as not requiring an increase in mechanical complexity or added mass. Electronic solutions are low in cost and have the advantage of being able to be located where it is convenient, not necessarily where dictated by good mechanical design.

The added electrical damping is also dynamically controllable, enabling it to be added when needed and disable when not needed. Future work could address a frequency dependent active damping, since added mechanical dissipation is rarely needed at low frequency [3].

The maximum level of added active electrical damping that can be implemented is limited by the stability of the circuit when in feedback with the motor amplifier. The level of damping is also relatively modest at this point, compared to the damping that can be generated with large mechanical dampers.

\section{ACKNOWLEDGEMENTS}

This work was supported in part by Northwestern University, National Science Foundation IGERT Fellowships through grant DGE9987577, and National Science Foundation Grant No. 0413204.

\section{REFERENCES}

[1] R. J. Adams and B. Hannaford. Stable haptic interaction with virtual environments. IEEE Transactions on Robotics and Automation, 15:465-474, 1999.

[2] R. J. Adams, M. R. Moreyra, and B. Hannaford. Stability and performance of haptic displays: Theory and experiments. In Proceedings ASME International Mechanical Engineering Congress and Exhibition, pages 227-234, Anaheim, CA, 1998. ASME.

[3] J. M. Brown. A theoretical and experimental investigation into the factors affecting the z-width of a haptic display. Master's thesis, Northwestern University, Evanston, IL, March 1995.

[4] J. E. Colgate and J. M. Brown. Factors affecting the z-width of a haptic display. In IEEE International Conference on Robotics and Automation, volume 4, pages 3205-3210, San Diego, CA, 1994. IEEE.

[5] J. E. Colgate and G. G. Schenkel. Passivity of a class of sampled-data systems: Application to haptic interfaces. Journal of Robotic Systems, 14(1):37-47, 1997.

[6] N. Diolaiti and G. Niemeyer. Wave haptics: Providing stiff coupling to virtual environments. In IEEE Symposium on Haptic Interfaces, volume 4, pages 185-192, Alexandria, VA, 2006. IEEE.

[7] R. B. Gillespie and M. R. Cutkosky. Stable user-specific haptic rendering of the virtual wall. In ASME International Mechanical Engineering Conference and Exposition, DSC, volume 58, pages 397-406, Atlanta, GA, 1996. ASME.

[8] M. Kawai and T. Yoshikawa. Haptic display of movable virtual object with interface device capable of continuous-time impedance display by analog circuit. In IEEE International Conference on Robotics and Automation, pages 229-234, Washington, DC, 2002. IEEE.

[9] M. Kawai and T. Yoshikawa. Haptic display with an interface device capable of continuous-time impedance display within a sampling period. IEEE/ASME Transactions on Mechatronics, 9(1):58-64, 2004.

[10] K. J. Kuchenbecker, J. Fiene, and G. Niemeyer. Improving contact realism through event-based haptic feedback. IEEE Transactions on Visualization and Computer Graphics, 12:219-230, 2006.

[11] D. A. Lawrence, L. Y. Pao, A. M. Dougherty, M. A. Salada, and Y. Pavlou. Rate-hardness: a new performance metric for haptic interfaces. IEEE Transactions on Robotics and Automation, 16:357-371, 2000.

[12] J. S. Mehling, J. E. Colgate, and M. A. Peshkin. Increasing the impedance range of a haptic display by adding electrical damping. In IEEE First World Haptics Conference and Symposium, pages 257262, Pisa, Italy, 2005. IEEE.

[13] A. M. Okamura, M. R. Cutkosky, and J. T. Dennerlein. Improving reality-based models for vibration feedback. IEEE/ASME Transactions on Mechatronics, 6(3):245-252, 2001.

[14] D. W. Weir, M. A. Peshkin, J. E. Colgate, and P. Buttolo. Design and performance of a high fidelity, low mass, linear haptic display. In IEEE First World Haptics Conference and Symposium, pages 177-182, Pisa, Italy, 2005. IEEE. 